\title{
The NPOESS Preparatory Project Science Data Segment: Brief Overview
}

\author{
Robert J. Schweiss, Evelyn Ho, Richard Ullman, Shahin Samadi \\ NASA Goddard Space Flight Center, Code 429 \\ Greenbelt, MD, USA \\ robert.j.schweiss@nasa.gov, evelyn.1.ho@nasa.gov, richard.e.ullman@nasa.gov, shahin.samadi@gsfc.nasa.gov
}

\begin{abstract}
The NPOESS Preparatory Project (NPP) provides remotely-sensed land, ocean, atmospheric, ozone, and sounder data that will serve the meteorological and global climate change scientific communities while also providing risk reduction for the National Polar-orbiting Operational Environmental Satellite System (NPOESS), the U.S. Government's future low-Earth orbiting satellite system monitoring global weather and environmental conditions. NPOESS and NPP are a new era, not only because the sensors will provide unprecedented quality and volume of data but also because it is a joint mission of three federal agencies, NASA, NOAA, and DoD. NASA's primary science role in NPP is to independently assess the quality of the NPP science and environmental data records. Such assessment is critical for making NPOESS products the best that they can be for operational use and ultimately for climate studies. The Science Data Segment (SDS) supports science assessment by assuring the timely provision of NPP data to NASA's science teams organized by climate measurement themes. The SDS breaks down into nine major elements, an input element that receives data from the operational agencies and acts as a buffer, a calibration analysis element, five elements devoted to measurement based quality assessment, an element used to test algorithmic improvements, and an element that provides overall science direction. This paper will describe how the NPP SDS will leverage on NASA experience to provide a mission-reliable research capability for science assessment of NPP derived measurements.
\end{abstract}

National Polar-orbiting Operational Environmental Satellite System(NPOESS); NPOESS Preparatory Project (NPP) Science Data Segment; leverage off existing science systems; climate studies; environmental data records; quality assessment; NASA; NOAA;DoD

\section{INTRODUCTION}

The NPP Science Data Segment is a prototype element for future Earth Science Enterprise (ESE) distributed data systems. The mission of ESE is to develop a scientific understanding of the Earth system and its responses to changes, as well as to improve prediction capabilities for climate, weather, air quality and natural hazards. The SDS is intended to be a research tool that uses a fully distributed interoperable architecture, with independent elements organized around key Environmental Data Records: atmospheric sounding, ocean, land, ozone, and atmospheric composition products. The SDS is intended to enable Climate Analysis Research Systems (CARS) development that will focus on the following areas: Atmospheric Composition, Climate Change,
Carbon/Ecosystems, Solid Earth, Weather, and Water/Energy Cycle. [2]

The primary role of the NPP SDS is limited to assessing the quality of the NPP Environmental Data Records (EDRs) for accomplishing climate research. In instances where EDRs are short of supporting climate research, algorithm enhancements can be provided and demonstrated. The NPP SDS is preparing for a September 2009 Launch Readiness Date and completed it's system requirements review in April 2005.

\section{BACKGROUND}

The National Polar-orbiting Operational Environmental Satellite System (NPOESS) is the U.S. Government's future low-earth orbiting weather and environmental satellite system, monitoring global weather and environmental systems. NPOESS and NPOESS Preparatory Project (NPP) are a new era, not only because the sensors will provide unprecedented quality and volume of data, but also because it is a joint mission of three federal agencies: National Aeronautic and Space Administration (NASA), Department of Defense (DoD), and the National Oceanic Atmospheric Administration (NOAA) [5]. NPP provides a "bridge" between current and future missions by providing pre-operational on-orbit test and risk reduction for key NPOESS instruments and ground-based data processing capabilities, while maintaining continuity of environmental data used for long-term climate change research.

The NPP mission provides remotely sensed land, ocean, atmospheric, ozone, and sounder data that will serve the meteorological and global climate change scientific communities while also providing risk reduction for NPOESS. The NPP system is defined at the highest level in terms of segments, one of which is the Science Data Segment (SDS). The NPP Science Data Segment is a prototype element for discipline-based processing. In this case, the SDS is intended to be a research tool and as such has no nominal operational requirements. Therefore, it is essential that the SDS maximize its interoperability with other Earth Science Enterprises (ESE) data system components. The operational requirements are met by two segments of the NPOESS/NPP systems: the Interface Data Processing Segment (IDPS) and the Archive and Distribution Segment (ADS), more commonly known as the NOAA CLASS [6]. The role of the SDS is limited to assessing the quality of the NPP Environmental Data Records (EDRs), and indirectly the Sensor Data Records (SDRs), for 
accomplishing climate research and providing algorithm enhancements when possible.

\section{SYSTEM DESCRIPTION}

The SDS is composed of the following nine elements: the SDS Data Distribution and Depository Element (SD3E), the Integration and Test System Element (I\&TSE), the Project Science Office Element (PSOE), the NPP Instrument Calibration Support Element (NICSE), and five Product Evaluation and Analysis Tools Elements (PEATEs), one for each of the following disciplines: Atmosphere, Land, Ocean, Ozone, and Sounder. A conceptual block diagram of the SDS is shown in Figure 1.

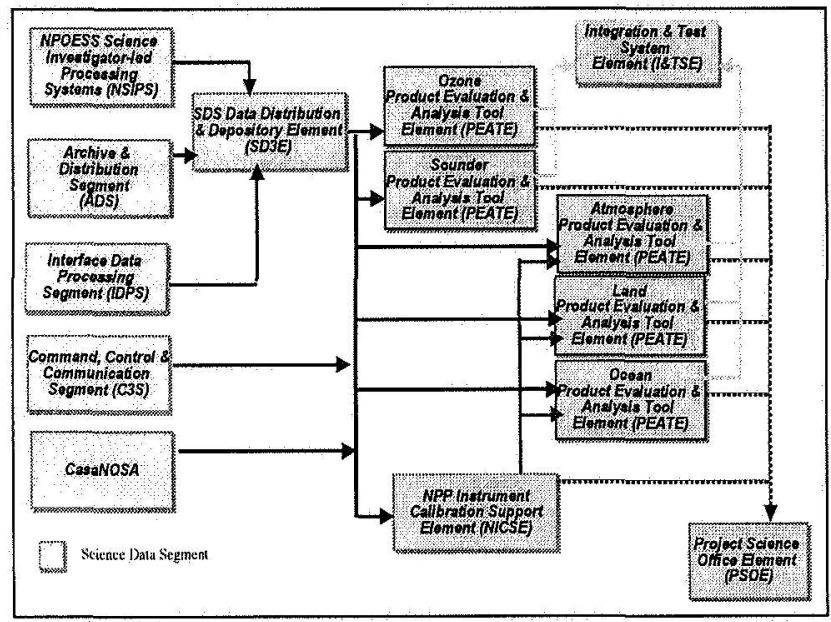

Fig. 1. SDS Conceptual Block Diagram

The SDS interfaces with the National Environment Satellite, Data, and Information Service (NESDIS) Interface Data Processing System (IDPS) primarily for receipt of RDRs and the NESDIS Comprehensive Large Array-data Stewardship System (CLASS) for receipt of select RDRs, SDRs, EDRs, and in some cases Temperature Data Records (TDRs). Additionally the SDS acquires, as needed, associated intermediate products, ancillary data, auxiliary data, operational algorithms, source code, documentation, or calibration coefficients from the CLASS. The NPOESS Command, Control, and Communications Segment (C3S) provides for the NPP satellite operation capabilities, communication, and routing of mission data to the ground receive stations. The SDS may request services from $\mathrm{C} 3 \mathrm{~S}$ by generating instrument service requests or engineering service requests. The Ancillary Data Providers (ADP) are the source for ancillary data products other than the NPOESS official ancillary data sets (e.g. Digital Elevation Models). In the capacity as Research tool the SDS works in concert with NASA Science Team Members. [1] The Science Teams are NASA-funded investigators from the broader science community, identified as contributors to the SDS by NASA Headquarters and the NPP Project Scientist. The Casa NOAA Observing System Architecture (NOSA) System serves as the NPOESS pre-launch data repository of data for distribution. The NPOESS System Investigator-led Processing System (NSIPS) serves as a temporary repository for the retained
Intermediate Products used for calibration and validation. The NPOESS/NPP Algorithm Configuration Management Review Board reviews the algorithm improvement recommendations for possible use in the operational environment.

\section{SDS INTERNAL ELEMENTS}

The SDS includes the following elements: the SD3E, a data ingest element that receives data from the operational segments and acts as a data storage buffer; the NICSE, an instrument calibration element for VIIRS; the PEATEs, five measurement based elements devoted to EDR quality assessment; the I\&TSE, an element used to test algorithmic improvements; and PSOE, an element that provides overall science direction.

\section{A. SDS Data Distribution and Depository Element}

The SDS Data Distribution and Depository Element (SD3E) serves as a central data buffer for the five Product Evaluation and Analysis Tool Element (PEATEs) and the NICSE by receiving data from the data providers, checking the integrity of the file, and staging the data for pick up by the PEATES and NICSE. Each of the PEATEs and NICSE submits their NPP data product request to the SD3E as either a subscription or as an ad-hoc request. For ad-hoc requests, the PEATEs and NICSE submit their orders to the SD3E on an as needed basis. For example, if the PEATEs or NICSE find problems with a particular data file (e.g. file errors) that warrants a re-request and a retransmission of the file, the SD3E submits the ad-hoc request to the appropriate data provider. For subscription orders, the SD3E compiles all six requests and submits one request to the NESDIS CLASS or IDPS using the appropriate interface mechanism designated by the data provider. If a PEATE is interested in data beyond the 32 day storage window of the SD3E then that element my go directly to the NOAA CLASS. The centralized approached eliminates duplicative data subscriptions and transfers by the NESDIS CLASS and or IDPS to each PEATE. This significantly reduces the data bandwidth from approximately $18 \mathrm{~TB}$ to $3 \mathrm{~TB}$ per day. The SD3E is sized for approximately 32 days.

\section{B. Integration and Test System Element}

The Integration and Test System Element (I\&TSE) provides the five PEATEs, the NICSE, and the science investigation teams a means for demonstrating algorithm or calibration enhancements, diagnosing science quality anomalies, and if necessary capability of regenerating intermediate products. The I\&TSE is a much smaller scale IDPS consisting of the same hardware environment as the production systems.

\section{Project Science Office Element}

The Project Science Office Element (PSOE) provides the overall science direction to the SDS PEATEs. The project science office provides management direction as well as mission and science guidance. This element also reviews the algorithm and calibration recommendations, made by the PEATEs and NICSE, prior to submitting them to the NPOESS/NPP Algorithm Configuration Management Review 
Board for review. Additionally, the PSOE may request volume and data transfer reports from the SD3E.

\section{NPP Instrument Calibration and Science Element}

The NPP Instrument Calibration and Science Element (NICSE) assesses and validates pre-launch and post-launch radiometric and geometric calibration and characterization of the VIIRS instrument data. The NICSE evaluates the calibration products, and if necessary, provides recommendations for calibration software and look-up table enhancements. The NICSE works closely with the Land, Atmosphere, and Ocean PEATEs to analyze data sets, harvest calibration information, test possible calibration changes, and validate calibration recommendations. NICSE may use the I\&TSE to validate that the recommended updates produce the expected results. Once changes have been validated, the calibration updates may be submitted to the PSOE for review and further action.

\section{E. Product Evaluation and Analysis Tool Element (PEATE)}

The five selected PEATEs are: Atmosphere, Land, Ocean, Ozone, and Sounder. Each of the elements is leveraged off of an existing system with the resources, the expertise, and the knowledge of the science of their respective discipline. Ocean PEATE is leveraged of existing resources from the Seaviewing Wide Field-of-view Sensor (SeaWiFS) Ocean Data Processing System (ODPS), which is a science investigator-led processing system that processes, calibrates, validates, archives, and distributes data received from the SeaWiFS instrument aboard the OrbView-2 observatory. The Land PEATE leverages off of the Moderate Resolution Imaging Spectroradiometer (MODIS) Data Processing System (MODAPS). MODAPS currently generates Level 2 through Level 4 MODIS science products for distribution to the Distributed Active Archive Centers (DAACs) for archival and to the MODIS science team for quality control. Ozone PEATE assess products with OMIDAPS [3], an existing system that processes data from the Ozone Monitoring Instrument (OMI) instrument aboard the Aura satellite to higher-level science data products. The Atmosphere PEATE will leverage scientific expertise at the Space Science and Engineering Center (SSEC) from the University of Wisconsin-Madison, presently, among other things, routinely validating the GOES Imager and Sounder products. The Sounder PEATE leverages off of the Atmospheric Infrared Sounder (AIRS) Project Team Leader Science Computing Facility (TLSCF) located at NASA/Jet Propulsion Laboratory currently ingesting AIRS, AMSU and HSB Level 0 data from the GSFC DAAC and operationally produces Level $1 \mathrm{~B}^{1}$ products. Figure 2 is the list of EDRs to be evaluated and their respective assignment to each SDS PEATE.

In the pre-launch time frame each of the PEATEs acquire, adapt and integrate science and operational SDR and EDR software into processing systems, adapt and update existing systems, perform functional testing of operational code, acquire and manage various preflight instrument characterization data

\footnotetext{
${ }^{1}$ RDR $=$ Raw Data Record $\sim$ CEOS/NASA Level $1 \mathrm{~A}$

$\mathrm{SDR}=$ Sensor Data Record $\sim$ CEOS/NASA Level IB

$\mathrm{EDR}=$ Environmental Data Record $\sim$ CEOS/NASA Level 2
}

sets, and support, as necessary, compatibility and functional testing. Additionally, as needed, support generation and review of proxy and simulated data and provide independent review of critical program information such as end user data formats.

During the post launch era, nominally, each PEATE acquires respective RDR, SDR, \& EDR, data sets of interest from the SD3E. After integrity verification data is then cataloged and archived. The data is then validated ${ }^{2}$ against ground-base or in situ measurements. On-orbit instrument performance and calibration are assessed (e.g. detector striping). Select RDRs are processed to SDRs \& EDRs using adapted or wrapped production software with alternative calibration parameters, and SDRs are processed to EDRs using revised or alternative algorithms. The production EDRs and the locally generated EDRs are made available internally to respective Science Team members for further analysis including, Cross-Comparison with concurrent observations, comparison with past data sets from other missions, assessments of internal consistencies and effect of flagging and masking algorithms.

Extracting production software and wrapping it with software, i.e., wrappers, encapsulates the production SDR EDR algorithm such that it is more easily used out side the production environment. The original production code is unchanged. This permits the PEATEs to fully evaluate the science algorithms as implemented in the operational environment and diagnose problems that may surface during the evaluation and analysis of the products. Additionally wrapping the production affords the SDS PEATES the ability to develop and test corrections and enhancements to the operational code that may ultimately lead to algorithm improvement recommendations.

The Ozone PEATE will provide support in assessing and validating pre-launch and post-launch radiometric calibration and characterization of OMPS instrument data.

The Sounder PEATE will provide support in assessing and validating pre-launch and post-launch radiometric calibration and characterization of ATMS and CrIS instrument data.

\begin{tabular}{|ll}
\hline LAND PEATE & Atmosphere PEATE \\
1. Albedo (Surface) & 13. Suspended Matter \\
2. Land Surface Temperature & 14. Cloud Cover/Layers \\
3. Snow Cover and Depth & 15. Cloud Effective Particie Size \\
4. Surface Type & 16. Cloud Top Height \\
5. Net Heat Flux & 17. Cloud Top Pressure \\
6. Ice Surface Temp. & 18. Cloud Top Temperature \\
7. Vegetation index & 19. Cloud Base Height \\
8. Aerosol Optical Thickness & 20. Cloud Optical Thickness \\
9. Aerosol Particle Size & Solnder PEATE \\
Ocean PEATE & 21. Almospheric Vertical Moisture \\
10. Ocean Color/Chlorophyl & Profile \\
11. Sea Surface Temperature & 22. Almospheric Vertical \\
Ozone PEATE & Temperature Profile \\
12. Ozone Total ColumniProfile & \\
Figure 2. EDR as allocated to SDS Elements
\end{tabular}

\footnotetext{
2 The validations steps don't apply to all PEATEs.
} 


\section{CONCLUSION}

NPP is a system that marks a change from internal data management during mission lifetime, to an approach that relies on heavy collaboration with operational agencies to manage initial processing and to perform data archive and distribution. The NPP SDS is built based on leveraging off of existing data processing centers to meet its requirement of data quality assessment of climate data products. Using the resources of existing systems eliminates the need to build entire data systems from scratch. These systems afford the NPP SDS the ability to tap the knowledge and the experience of the science investigators, lessons learned from building previous data systems, their expertise in climate data analysis, reuse of existing data systems, and reduction in system development costs. As a result, this collaboration of five fully functionally independent elements enables the NPP SDS to meet its requirements in accomplishing climate research.
We thank the members of the NPP SDS team for their contributions to this work. Additionally, information from various NPP documents were used and presented.

\section{REFERENCES}

[1] NRA-03-OES-01, Research Announcement, NPP Science Team for Climate Data Records, January 28, 2003

[2] Asrar, Ghassem, Level I Requirements for the NPOESS Preparatory Project (NPP), Signed November 3, 2003

[3] Tilmes, C., Linda, M., Fleig, A., 2004, Development of two Science Investigator-led Processing Systems (SIPS) for NASA's Earth Observation System (EOS), IEEE Geoscience and Remote Sensing Society, 2004

[4] Krimchansky, S., Performance for First Flight Unit of the Advanced Technology Microwave Sounder (ATMS), Proc. of SPIE Vol. 5979, 597926, doi: $10.1117 / 12.632429,2005$

[5] NOAA Satellite and Information Service, National Environmental Satellite, Data and Information Service (NESDIS), National Polarorbiting Operational Environmental Satellite System (NPOESS), Retrieved April 20, 2006, from http:/www.ipo.noaa.gov

[6] NOAA Satellite and Information Service, National Environment Satellte, Data, and Information Service (NESDIS), Retrieved April 20, 2006, from http://www.osd.noaa.gov/class/ 